\title{
$\left[\mathrm{Cu}(\mathrm{NTO})_{2}\left(\mathrm{H}_{2} \mathrm{O}\right)_{2}\right] \cdot 2 \mathrm{H}_{2} \mathrm{O}$ 的制备、 分子结构和热分解机理
}

张同来* 胡荣祖** 李福平陈里 (西安近代化学研究所, 西安 710061)

\author{
郁 开 北 \\ (中国科学院成都分院, 成都 610041)
}

\section{关链调 NTO、铜（II）配合物、分子结构、热分解机理}

3-硝基-1,2,4-三坐-5-酮 (NTO) 作为一种高能低感度含能材料受到了有关工作者 的极 大重视 ${ }^{[1,2]}$. 其金属盐在火炸药方面也有很重要的用途, 因而引起了人们很大的兴趣 ${ }^{[3,4]}$. 我们制 备了 NTO 的铜 (II) 配合物的单晶,测定了其结构,并研究了它的热分解机理.

\section{1 实验部 分}

\section{1 样品制备}

将 NTO 分散于蒸馏水中 $(\mathrm{M}: \mathrm{V}-1: 4)$. 在 $60^{\circ} \mathrm{C}$ 下, 边搅拌边用氢氧化锂的水溶液中 和至 $\mathrm{pH}$ 值 7 左右. 然后将制得的溶液在 $60^{\circ} \mathrm{C}$.下逐渐滴加到硫酸铜水溶液中, 得到淡蓝色 沉淀物. 将沉淀物溶于蒸馏水中, 在室温下培养得到用于 $\mathrm{x}$ 光结构测定的深蓝色单晶. 元素 分析结果,计算值(\%): Cu 15.98, C 12.20,N 28.46, H 2.56; 实测值(\%): Cu 15.11, C 11.89, $\mathrm{N} 28.35, \mathrm{H} 2.49$. IR: $\nu_{\mathrm{C}=\mathrm{O}}-1651 \mathrm{~cm}^{-1}, \nu_{\mathrm{NO}}^{\text {ss }}-1529 \mathrm{~cm}^{-1}, \nu_{\mathrm{NO}_{2}}^{\mathrm{s}}-1319 \mathrm{~cm}^{-1}, \nu_{\mathrm{M}-\mathrm{O}}-455$ $\mathrm{cm}^{-1}, \nu_{M-N}-321 \mathrm{~cm}^{-1}$.

\section{2 晶体结构测定}

选用尺寸为 $0.20 \times 0.38 \times 0.48 \mathrm{~mm}^{3}$ 的单晶, 在 Nicolet $\mathrm{R} 3 \mathrm{M} / \mathrm{E}$ 型四圆衍射仪上, 用 $\mathrm{MoK}_{\alpha}$ 射线、石墨单色器、 $\lambda-0.71073 \AA$, 在 $5^{\circ}<\theta<14^{\circ}$, 用 25 个衍射点精确测定取向矩阵 和晶胞参数. 用 $\theta / 2 \theta$ 方式扫描, 在 $10^{\circ}<\theta<35^{\circ}$ 范围内共收集独立衍射点 3097 个, 其中 $I>3.0 \sigma(I)$ 者为 2765 个用于结构测定和修正, 所得晶体学参数为: $a-6.458(1) \AA, b=$ $6.633(1) \AA, c-8.115(1) \AA, a-105.63(1)^{\circ}, \beta-99.99(1)^{\circ}, \gamma=94.81(1)^{\circ}, V-326.59(9)$ $\AA^{3}, Z-1, D_{\mathrm{c}}-2.00 \mathrm{~g} \cdot \mathrm{cm}^{-3}, \mu-17.47 \mathrm{~cm}^{-1}, F(000)-199$. 晶体属三斜晶系, 空间点群 $P-1$. 数据经 Lp 校正, 但末经吸收校正, 对两个标准反射的强度监测表明强度无重大变 化.

Cu 原子坐标由直接法解出, 其它原子坐标由差值 Fourier 合成法得到. 结构由块矩阵 最小二乘法优化, 除氢原子采用各向同性热参数外, 其它原子均采用各向异性热参数, 最终偏

1992-07-15 收稿, 1992-10-30 收修改稿.

* 华东工学院博士研究生.

* 联系人. 
表 1 原子坐标 $\left(\times 10^{4}\right)$ 和等效温度因子 $\left(\AA^{2} \times 10^{3}\right)$

\begin{tabular}{|c|c|c|c|c|}
\hline 原 子 & $x$ & $y$ & 8 & $U_{\bullet Q}$ \\
\hline $\mathrm{Cu}$ & 0 & 0 & 0 & $\left.19(1)^{2}\right)$ \\
\hline$N(1)$ & $-3224(2)$ & $3656(2)$ & $-2644(1)$ & $28(1)^{n)}$ \\
\hline $\mathrm{N}(2)$ & $-3067(2)$ & $2009(2)$ & $-4029(1)$ & $29(1)^{4)}$ \\
\hline$N(3)$ & $-1059(2)$ & $1428(2)$ & $-1477(1)$ & $22(1)^{4)}$ \\
\hline$N(4)$ & $-1607(2)$ & $-1232(2)$ & $-4189(2)$ & $28(1)^{21}$ \\
\hline$\phi(1)$ & $-2326(2)$ & $4565(2)$ & $405(1)$ & $29(1)^{01}$ \\
\hline$O(2)$ & $-2025(2)$ & $-1733(2)$ & $-5787(2)$ & $41(1)^{21}$ \\
\hline$O(3)$ & $-754(2)$ & $-2299(2)$ & $-3302(2)$ & $40(1)^{a)}$ \\
\hline$O(4)$ & $-2646(2)$ & $-1472(2)$ & $275(1)$ & $28(1)^{n}$ \\
\hline $\mathrm{O}_{\mathrm{w}}$ & $4251(2)$ & $6942(2)$ & $7288(1)$ & $32(1)^{4}$ \\
\hline$c(1)$ & $-2393(2)$ & $3340(2)$ & $-1076(2)$ & $22(1)^{4}$ \\
\hline$C(2)$ & $-2125(2)$ & $770(2)$ & $-3240(1)$ & $23(1)^{4}$ \\
\hline$H(4 a)$ & $-3638(37)$ & $-1687(39)$ & $-553(30)$ & $36(5)$ \\
\hline$H(4 b)$ & $-2594(44)$ & $-2617(38)$ & $428(36)$ & $44(6)$ \\
\hline $\mathrm{H}$. & $-3999(39)$ & $4672(38)$ & $-2750(34)$ & $41(6)$ \\
\hline $\mathrm{H}_{\mathrm{wa}}$ & $3273(43)$ & $6458(45)$ & $7624(38)$ & $50(7)$ \\
\hline $\mathrm{H}_{\boldsymbol{\nabla b}}$ & $3596(50)$ & $7429(51)$ & $6549(39)$ & $62(8)$ \\
\hline
\end{tabular}

8) 各问同性当骨: $U_{0 q}=\frac{1}{3}\left(U_{11}+U_{22}+U_{33}\right)$.

表 2 化学键长 $(\boldsymbol{A})$

\begin{tabular}{l|l|l|l}
\hline $\mathrm{Cu}-\mathrm{N}(3)$ & $1.954(1)$ & $\mathrm{Cu}-\mathrm{O}(4)$ & $1.973(1)$ \\
$\mathrm{Cu}-\mathrm{N}(3 \mathrm{~s})$ & $1.954(1)$ & $\mathrm{Cu}-\mathrm{O}(4 \mathrm{a})$ & $1.973(1)$ \\
$\mathrm{N}(1)-\mathrm{N}(2)$ & $1.365(2)$ & $\mathrm{N}(1)-\mathrm{C}(1)$ & $1.370(2)$ \\
$\mathrm{N}(1)-\mathrm{H}$ & $0.886(27)$ & $\mathrm{N}(2)-\mathrm{C}(2)$ & $1.299(2)$ \\
$\mathrm{N}(3)-\mathrm{C}(1)$ & $1.368(2)$ & $\mathrm{N}(3)-\mathrm{C}(2)$ & $1.349(1)$ \\
$\mathrm{N}(4)-\mathrm{O}(2)$ & $1.223(2)$ & $\mathrm{N}(4)-\mathrm{O}(3)$ & $1.235(2)$ \\
$\mathrm{N}(4)-\mathrm{C}(2)$ & $1.442(2)$ & $\mathrm{O}(1)-\mathrm{C}(1)$ & $1.247(1)$ \\
$\mathrm{O}(4)-\mathrm{H}(4 \mathrm{a})$ & $0.816(22)$ & $\mathrm{O}(4)-\mathrm{H}(4 \mathrm{~b})$ & $0.804(27)$ \\
$\mathrm{Ow}-\mathrm{Hwa}$ & $0.806(31)$ & $\mathrm{Ow}-\mathrm{Hwb}$ & $0.826(35)$ \\
\hline
\end{tabular}

表3 键角 $\left({ }^{\circ}\right)$

\begin{tabular}{l|l|l|r}
\hline $\mathrm{N}(3)-\mathrm{Cu}-\mathrm{O}(4)$ & $90.0(1)$ & $\mathrm{N}(3)-\mathrm{Cu}-\mathrm{N}(3 \mathrm{a})$ & $180.0(1)$ \\
$\mathrm{O}(4)-\mathrm{Cu}-\mathrm{N}(3 \mathrm{a})$ & $90.0(1)$ & $\mathrm{N}(3)-\mathrm{Cu}-\mathrm{O}(4 \mathrm{a})$ & $90.0(1)$ \\
$\mathrm{O}(4)-\mathrm{Cu}-\mathrm{O}(4 \mathrm{a})$ & $180.0(1)$ & $\mathrm{N}(2)-\mathrm{N}(1)-\mathrm{C}(1)$ & $112.1(1)$ \\
$\mathrm{N}(2)-\mathrm{N}(1)-\mathrm{Hn}$ & $123.0(16)$ & $\mathrm{C}(1)-\mathrm{N}(1)-\mathrm{Hn}$ & $123.8(17)$ \\
$\mathrm{N}(1)-\mathrm{N}(2)-\mathrm{C}(2)$ & $101.4(1)$ & $\mathrm{Cu}-\mathrm{N}(3)-\mathrm{C}(1)$ & $131.1(1)$ \\
$\mathrm{Cu}-\mathrm{N}(3)-\mathrm{C}(2)$ & $124.8(1)$ & $\mathrm{C}(1)-\mathrm{N}(3)-\mathrm{C}(2)$ & $103.7(1)$ \\
$\mathrm{O}(2)-\mathrm{N}(4)-\mathrm{O}(3)$ & $125.3(1)$ & $\mathrm{O}(2)-\mathrm{N}(4)-\mathrm{C}(2)$ & $118.3(1)$ \\
$\mathrm{O}(3)-\mathrm{N}(4)-\mathrm{C}(2)$ & $116.4(1)$ & $\mathrm{Cu}-\mathrm{O}(4)-\mathrm{H}(4 \mathrm{a})$ & $114.6(19)$ \\
$\mathrm{Cu}-\mathrm{O}(4)-\mathrm{H}(4 b)$ & $116.9(21)$ & $\mathrm{H}(4 \mathrm{a})-\mathrm{O}(4)-\mathrm{H}(4 b)$ & $103.4(26)$ \\
$\mathrm{Hwa}-\mathrm{Ow}-\mathrm{Hwb}$ & $100.0(32)$ & $\mathrm{N}(1)-\mathrm{C}(1)-\mathrm{N}(3)$ & $105.9(1)$ \\
$\mathrm{N}(1)-\mathrm{C}(1)-\mathrm{O}(1)$ & $126.4(1)$ & $\mathrm{N}(3)-\mathrm{C}(1)-\mathrm{O}(1)$ & $127.7(1)$ \\
$\mathrm{N}(2)-\mathrm{C}(2)-\mathrm{N}(3)$ & $117.0(1)$ & $\mathrm{N}(2)-\mathrm{C}(2)-\mathrm{N}(4)$ & $122.1(1)$ \\
$\mathrm{N}(3)-\mathrm{C}(2)-\mathrm{N}(4)$ & $120.9(1)$ & & \\
\hline
\end{tabular}


离因子 $R=0.0315, R_{w}=0.0320, S=1.205,\left(w-\left\{1 /\left[\sigma^{2}(F)+0.0003(F)^{2}\right]\right\} \times\{1-\exp [-\right.$ $\left.\left.\left.5(\sin \theta / \lambda)^{2}\right]\right\},(\Delta / \sigma)_{\max }-0.026\right)$, 最终差图上的最小高度为 -0.815 , 最大高度为 $1.051 \mathrm{e} \AA^{3}$ (由 Cu 原子起为 $0.75 \AA$ ). 晶体结构计算是用 SHELXTL 程序 (Sheldrick, 1985), 在 Eclipse $S / 140$ 计算机上进行的. 所得原子的坐标及温度因子、键长、键角分别列于表 1,2 和 3 , 该配合物的分子结构见图 1 , 晶体中配合物分子的堆积见图 2 .

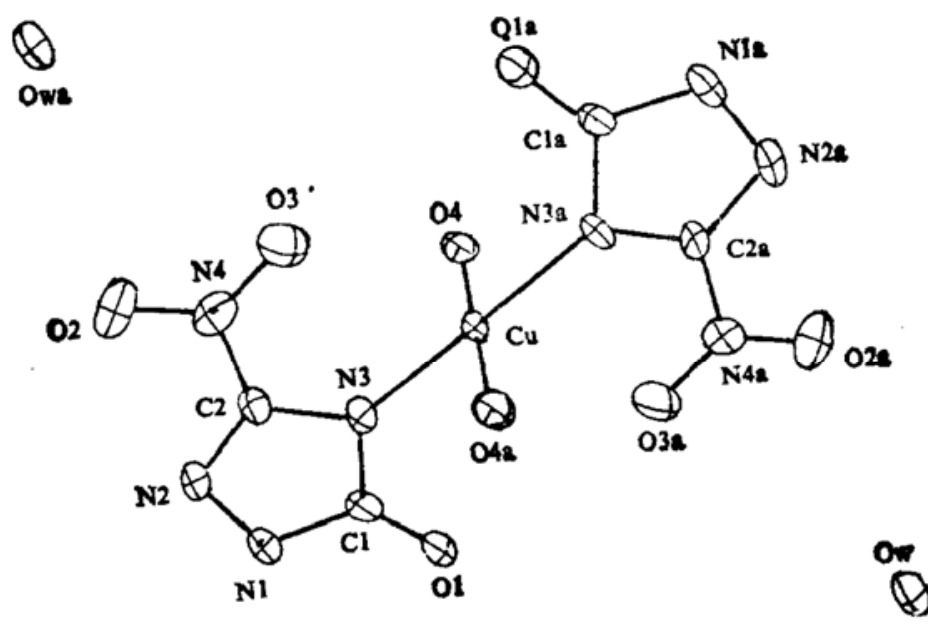

图 $1\left\lfloor\mathrm{Cu}(\mathrm{NTO})_{2}\left(\mathrm{H}_{2} \mathrm{O}\right)_{2}\right] \cdot 2 \mathrm{H}_{2} \mathrm{O}$ 的分子结构

\section{2 结果和讨论}

由结构分析结果可知, [Cu(NTO $\left.)_{2}\left(\mathrm{H}_{2} \mathrm{O}\right)_{2}\right] \cdot 2 \mathrm{H}_{2} \mathrm{O}$ 是具有中心对称的分子, $\mathrm{Cu}$ 原子位于 对称中心, 铜原子及其配位原子 $\mathrm{N}_{3}, \mathrm{~N}_{34}, \mathrm{O}_{4}$ 和 $\mathrm{O}_{42}$ 共平面, 其配位间距分别为 1.954(1), 1.954 (1), 1.973 (1) 和 1.973(1) $\AA$, 四个配位原子与 $\mathrm{Cu}$ 原子所形成的夹角均为 $90^{\circ}$, NTO 负 离子中的原子都处在一个平面 内, 但 两个 NTO 负离子不共面, 其两平面间距大约为 $0.37-0.38 \AA$, 两个 NTO 负离子所在的平 面与 $\mathrm{CuN}_{3} \mathrm{~N}_{34} \mathrm{O}_{4} \mathrm{O}_{42}$ 形成平面的二面角均为 $87.9^{\circ}$.

用 TG-DTG, DSC 和 FT-IR 技术对 其热分解机理研究结果为: 该配合物的 DSC 曲线在 $93-153^{\circ} \mathrm{C}$ 间有一吸热峰, 而 TGDTG 在相应的温度区间失重量为 $18.0 \%$,与 一次失去四个水分子的理 论值 $18.3 \%$ 相 符 合. 因此可以推断, [ $\left.\mathrm{Cu}(\mathrm{NTO})_{2}\left(\mathrm{H}_{2} \mathrm{O}\right)_{2}\right]$ -

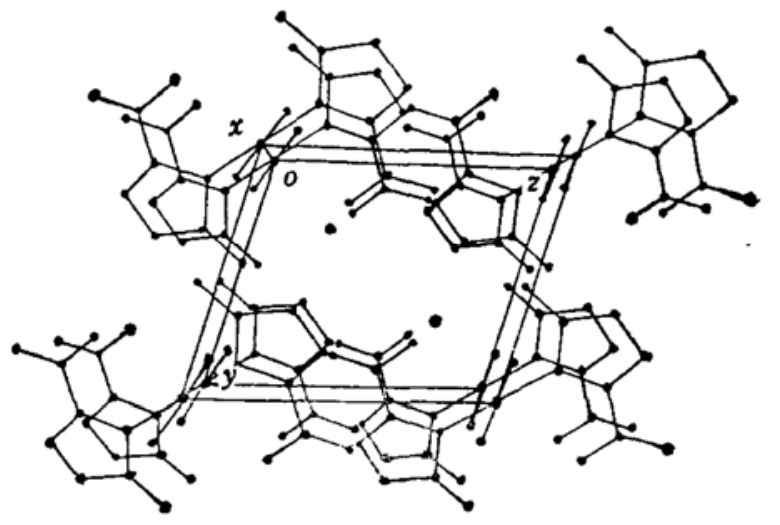

图 2 晶胞中 $\left[\mathrm{Cu}(\mathrm{NTO})_{2}\left(\mathrm{H}_{2} \mathrm{O}\right)_{2}\right] \cdot 2 \mathrm{H}_{2} \mathrm{O}$ 的分子堆积 $2 \mathrm{H}_{2} \mathrm{O}$ 的脱水过程是一步完成的. 由 $\mathrm{K}$ issinger 法求得的脱水活化能为 $119.0 \mathrm{~kJ} \cdot \mathrm{mol}^{-1}$. 脱 水后的物质很不稳定, 受热即进一步分解, 首先是一 $\mathrm{NO}_{2}$ 断裂离去, 随即三唑酮环破裂。230 ${ }^{\circ} \mathrm{C}$ 分解残渣的红外光谱在 2179 和 $1189 \mathrm{~cm}^{-1}$ 处的吸收谱带表明: 产物中有 $\mathrm{Cu}(\mathrm{NCO})_{2}$ 存在. 因 此可以推断: 三唑环是从 $C(2)-N(3)$ 和 $C(1)-N(1)$ 键处断裂的. 进一步的分解产物为 $\mathrm{CuCO}_{3}$, 再转变为 $\mathrm{CuO}$, 这就生成了对火药、推进剂的燃烧有催化作用的活性物质, 从而起到 了含能催化剂的作用. 


\section{参考文献}

[1] Lee, K. Y., Chapman, L. B., Coburn, M. D., J. Energetic Mater., 1987, 5:27-33.

[2] Rothgery, E. F., Audette, D. E., Wedlich, R. C. et al., Thermochim. Acta, 1991, 185:235-243.

[3] Redman, L. D., Spear, R. J., An Evalwation of Metal Salts of NTO as Potential Primary Explosives, AD-A220 339, 1990.

[4] Xie Yi. Hu Rong-zu, Wang Xi-you et al., Thermochim. Acıa, 1991, 189:283-296. 